\title{
Online short course for learning management system training in an African university of technology
}

\section{Leanri van Heerden}

Central University of Technology, Free State, Bloemfontein, South Africa.

\begin{abstract}
With the use of technology in the classroom growing every year, lecturers are expected to blend facilitations by making use of learning management systems. At the Central University of Technology, Free State this poses a problem for the learning designers in charge of training lecturers in educational technologies as there is limited space and time to not only teach lecturers the skills, but also to apply them in their own contexts. The purpose of this paper is to present participant perspectives of an online short course in which they learned how to use Blackboard and other educational technologies needed for blending teaching and learning. An exploratory design was implemented with descriptive statistics involving quantitative analysis of the collected data to determine the participants' perspective on their skills gained. An online survey tool was used to collect the data. Eightyfour percent of particpants felt that they were able to communicate via the system's announcements, $82 \%$ felt confident that they would be able to customize the space and $94 \%$ felt that they were now able to add content, such as files. When asked if they felt like they learned the skills they expected from the online course, $94 \%$ of particpants replied positively.
\end{abstract}

Keywords: e-learning; learning management system; Blackboard; instructional design; online course; educational technology. 


\section{Introduction}

The advancement of educational technology has created a need for lecturers to shift the classroom. Whether a course is completely online or a blend between online and face-toface (f2f), the benefits of a learning management system (LMS) in today's university environment are endless. At the Central University of Technology (CUT), Free State it is important for lecturers to master the LMS so that they may provide their students with the best facilitation possible. In an increasingly virtual world the need for rapid communication and continuous information flow is critical (Lee \& Owens, 2000) which means to prepare students for this new world the training of lecturers in all educational technology, especially the LMS is of paramount importance. Training of these lecturers come with a plethora of challenges that fall in the capable hands of the institution's e-learning team.

Traditionally the e-learning team at CUT would invite lecturers to f2f training sessions facilitated in a computer lab where participants can actively follow along. Two main issues stem from this approach. The first is that lecturers' time schedules are inconsistent and fixing an appropriate time that suits a large group of people is tricky. The second is that lecturers only learn the skills of the LMS at these sessions not how to apply them to their own contexts. The result being that lecturers do not benefit fully from these training sessions which leads to frustration and negativity toward the LMS. A means of training lecturers to not only use the tools of the LMS, but also learn how to apply those tools in their own contexts was necessary, but that was also convenient for all no matter their time schedule.

The question guiding this paper was focused on combining access and application. How can training of the LMS be conducted that benefits all? The aim was to develop an online short course where lecturers participate in their own time that not only facilitates the acquisition of skills, but also allows an experience of the online space as a student and apply the skills learned in a space provided where constructive feedback and peer learning can take place.

The purpose of this paper is to present perspectives of participants completing an online short course designed to be completed asynchronously with a space where new skills can be practiced. Literature underpinning the research will be discussed followed by the context of the study. The online course will be described, and participants' perspectives presented. Finally, the efficacy of this type of training in the higher education setting will be argued.

\section{Literature review}

F2F learning is when teaching and learning is facilitated while the lecturer and students share the same geographical location where in contrast, online learning occurs on a digital 
medium where parties work synchronously or asynchronously from anywhere. Blended learning is an approach that includes a combination of online and f2 $\mathrm{f}$ teaching and learning experiences. Blended learning will take place in both the classroom and online with the lecturer exercising some control over the time and pace of learning (Pullman \& Graham, 2018). To facilitate blended learning, an LMS can be used to host the online material and activities.

An LMS is an information system that facilitates e-learning (McGill \& Klobas, 2009) which means any teaching and learning experience that is supported by information technology. There are two main types of LMSs namely; commercial and open source (Beatty \& Ulasewicz, 2006). A commercial LMS will be distributed, hosted and supported by its owner company. An open source LMS is free to use, but the maintenance and support falls to the user. In the university setting, LMSs are used to create an online space where students can interact with content, lecturers and peers as well as online assessments and feedback. LMSs are generally managed by the Academic Support section, generally the elearning unit.

\section{Context}

In charge of support for academic staff at the CUT is the Centre for Innovation in Learning and Teaching (CILT). The purpose of CILT is to foster staff development and drive research in learning and teaching practice. There are five units within CILT of which eLearning and Educational Technology is the unit responsible for all e-learning and technology support on and off campus which includes managing and providing support for the LMS.

The CUT makes use of the commercial LMS software package, Blackboard Learn 9.1, distributed by Blackboard Inc. Training on the system is facilitated by the two learning designers and conducted $\mathrm{f} 2 \mathrm{f}$ in a computer lab with 20 or less participants at a time. The space limitation means multiple sessions must be set up to accommodate lecturers who have different schedules. Lastly, lecturers are not prone to immediately hone the new skills they learned at the training session as they did not learn to apply the skills in their contexts but merely to perform them. A more ideal method for training should not only teach the skills, but allow participants to actively apply them when it's still fresh as well as provide open access of the same training to all (Osuna-Acedo \& Gil-Quintana, 2017).

\section{Online course}

The purpose of the online course was to not only prepare lecturers for online delivery, but also to foster a network and culture of online learning on campus. 
The course covered the basics of Blackboard and at the end participants will be able to:

- Navigate the LMS interface by designing an effective user experience on Blackboard

- Communicate information to students by creating announcements, sending mails and facilitating discussion boards

- Build content by uploading files, creating learning activities and tool links

- Manage the LMS by enrolling students, creating calendar events and personalising settings.

The course took place over three weeks with the following being covered each week:

Table 1. Layout of online short course structure

\begin{tabular}{|c|c|c|}
\hline Week 1 & Week 2 & Week 3 \\
\hline $\begin{array}{l}\text { This week provides participants the } \\
\text { time to get used to the system and } \\
\text { explore some of the tools. During } \\
\text { this time everyone gets to know } \\
\text { each other on a shared digital pin- } \\
\text { up board (Padlet) and participate in } \\
\text { an ice breaker game in the } \\
\text { discussion forums. Participants then } \\
\text { personalize their settings by } \\
\text { updating their personal information } \\
\text { and uploading an avatar. The week } \\
\text { is wrapped up with a discussion on } \\
\text { making the online environment } \\
\text { friendlier for students. }\end{array}$ & $\begin{array}{l}\text { During Week } 2 \text { participants } \\
\text { enrol themselves into } \\
\text { groups and each group is } \\
\text { allocated a practice page } \\
\text { where they are enrolled as } \\
\text { instructors. They send } \\
\text { announcements and } \\
\text { organize the course menus } \\
\text { on the practice pages. Skills } \\
\text { learned during this week } \\
\text { also include using the } \\
\text { formatting palette to edit } \\
\text { type, embed images and } \\
\text { videos and link to websites. }\end{array}$ & $\begin{array}{l}\text { In Week } 3 \text { participants start } \\
\text { building content on their pages } \\
\text { as well as explore learning } \\
\text { theories for online delivery. } \\
\text { They use Google's Docs word } \\
\text { processing and Coggle } \\
\text { mindmap tools to create a } \\
\text { shared document with specific } \\
\text { outcomes and design a lesson } \\
\text { using Gagne's Steps of } \\
\text { Instruction respectively. }\end{array}$ \\
\hline
\end{tabular}

This course takes a very active hands-on approach and participants learn by doing as well as from peers during interactive discussion sessions. So particpants experienced an online course, hosted on the LMS exactly as their students would. This lets them learn as much by experience as from he instructor.

\section{Methodology}

An exploratory design was implemented involving quantitative descriptive analysis of the collected data to determine the participants' perspective on their skills gained during the online course. An exploratory design aims to develop insight into a phenomenon which is not well understood (De Vos et al., 2011), while a descriptive analysis requires that the 
researcher remains focused on what is given so that the experience of the phenomenon, as well as the structure of that experience can be discovered (Foster, 2016). An online survey tool, QuestionPro, was used to collect the data after each iteration was complete. Getting feedback from the participants of an online course is one of the success factors for online course delivery (Stevens, 2013). It allows instructional designers to hone best practices and gain insights from feedback to supplement other research results.

The target population of the study was the 63 participants who graduated one of the five iterations of the online course conducted between 2017 and 2018. For 3 weeks partipants completed daily activities that guided them to a new skill or tool every day. At the end of the 3-week period, graduates were encouraged to complete a feedback survey that focused on the participants' expectations of the course, whether those expectations were met, and the skills learned. The skills learned were determined by a five-point Likert scale that ranged from 1 star being The Horror! to 5 stars being Next Level Ninja! The skills questioned were aligned with those in the outcomes that were shared with participants at the commencement of the online course. Deeper knowledge was explored with questions regarding the participants' experience navigating the course, the usefulness of daily announcements and instructional videos and whether they would be able to incorporate their new skills into their daily practice.

Using the outcomes of the online course to guide the formulating of the questions strengthens the content validity of the questionnaire, ensuring the domain is adequately covered (Bolarinwa, 2015). Close- and open-ended questions were asked to maintain a balance between data that can be analysed, but also allowing deeper responses with freeform answers (De Vos et al., 2011).

\section{Results}

Thirty-three participants completed the questionnaire and their responses showed the following key results:

When asked if they felt like they learned the skills they expected from the online course, $94 \%$ of participants replied positively (70\% Absolutely and 24\% Most of the skills).

Participants were then asked about their perception of the level of the skills they learned by indicating in a Likert scale from 1 star being the lowest amount of skills attained and 5 stars being the highest. The questions were divided into the categories Communication, Navigation and Content Building for ease of presentation, with the former being discussed first.

Most of the participants, 84\%, felt that they achieved a high level (72\% 5 stars \& $12 \% 4$ stars) of skill when sending announcements via the LMS. Eighty-five percent of 
participants felt that they were able to send emails via the LMS (61\% 5 stars \& $24 \% 4$ stars). When participating in discussion boards, 79\% (49\% 5 stars \& $30 \% 4$ stars) felt that they have mastered the skill and 57\% (42\% 5 stars \& $15 \% 4$ stars) felt confident that they would be able to create and facilitate a discussion board.

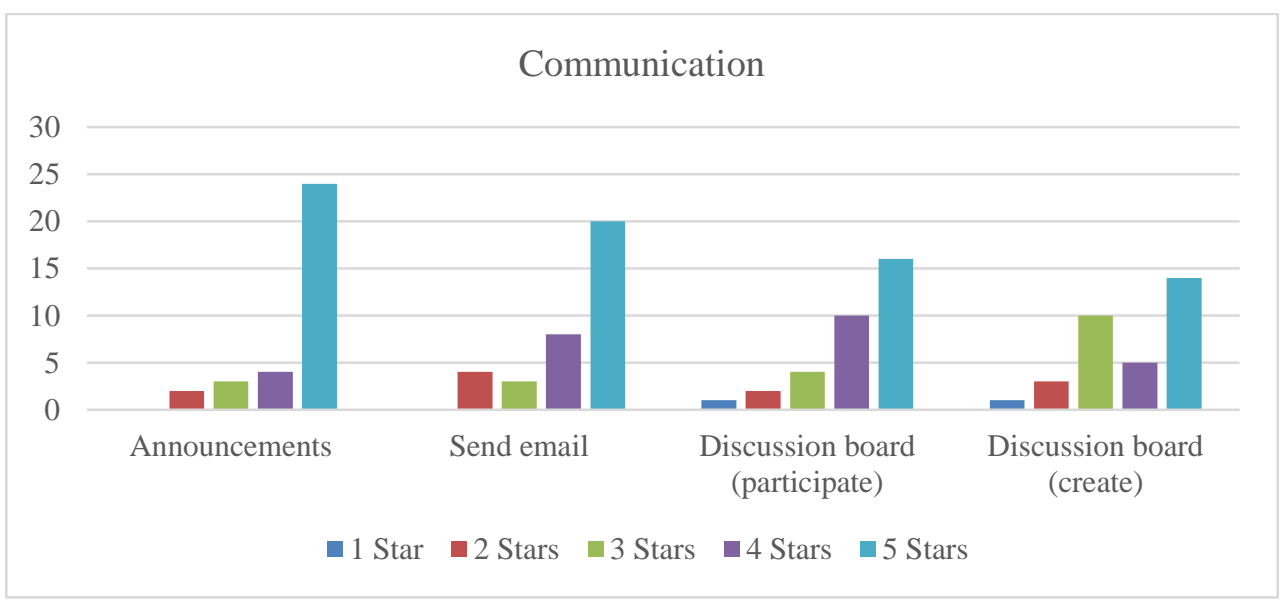

Figure 1. Participants' perspectives on level of communication skills gained

When focusing on navigational skills, the participants' responses indicated that $82 \%$ felt that they could confidently customize their personal settings on the LMS (64\% 5 stars \& $18 \% 4$ stars) and $63 \%$ (42\% 5 stars \& $21 \% 4$ stars) felt comfortable working with the groups feature. When exploring the content editor $85 \%$ of participants felt that they have got the skills to use this tool beneficially ( $61 \% 5$ stars \& $24 \% 4$ stars).

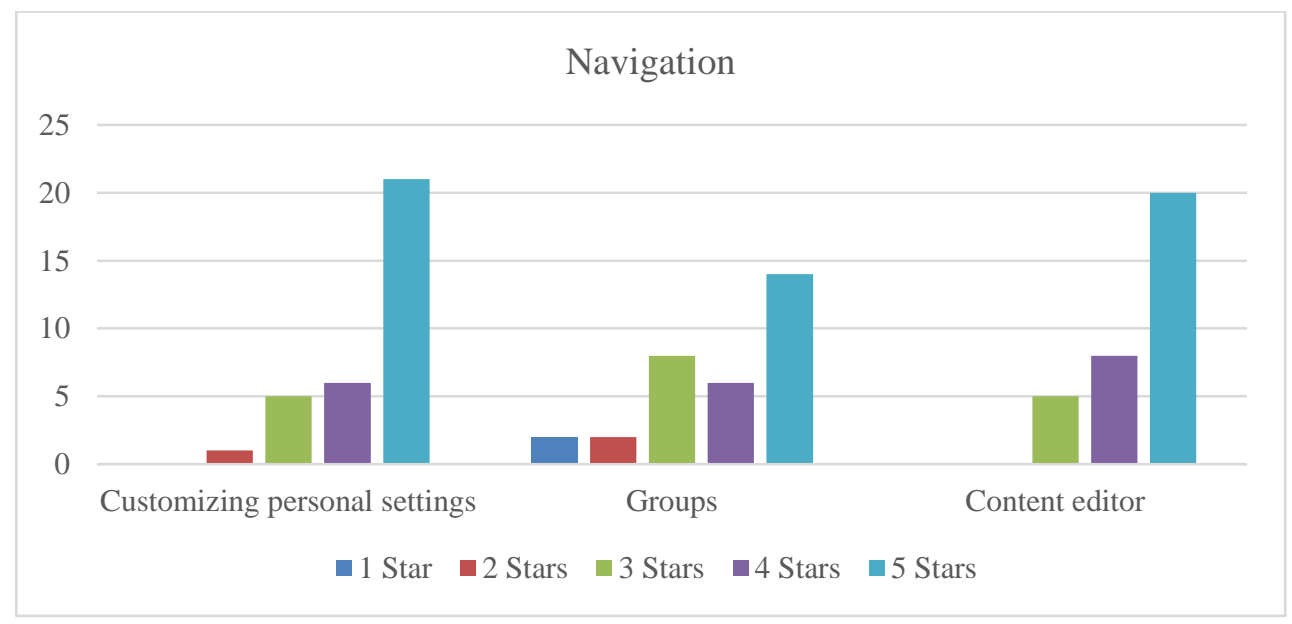

Figure 2. Participants' perspectives on level of navigation skills gained 
During the content building skills section, participants felt very positive toward adding files (94\%), images (85\%) and videos (82\%). The final skill, blogs, received a more lukewarm response as only $27 \%$ of participants selected 5 stars and $30 \% 4$ stars. Although $57 \%$ still indicates a majority it is perceptibly less than the other skills.

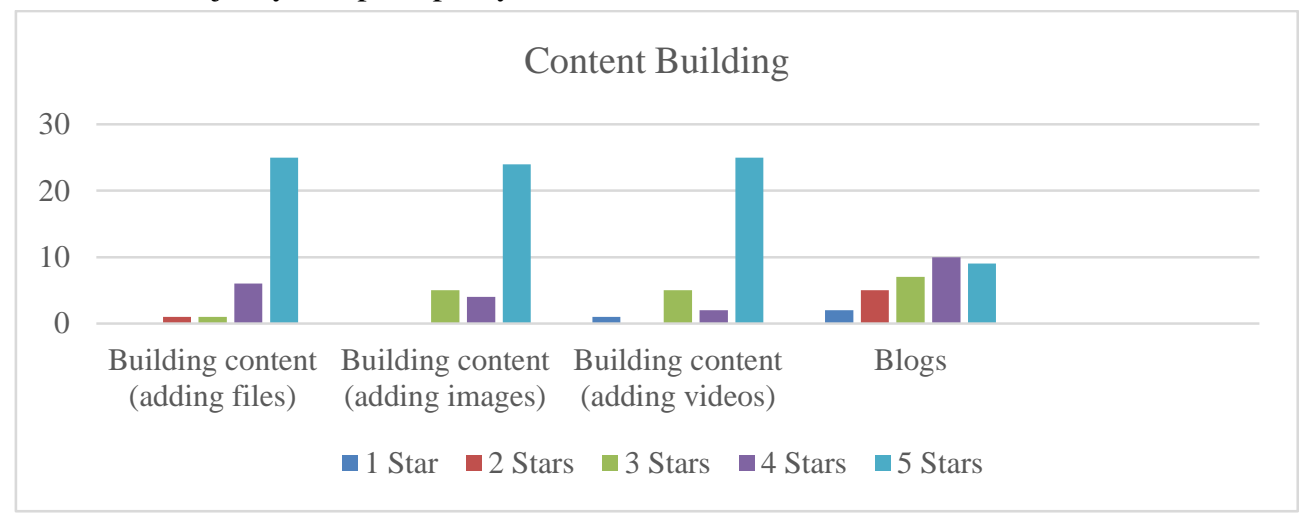

Figure 3. Participants' perspectives on level of content building skills gained

Participants were then asked if they felt they would be able to use the new skills in their teaching and learning practice. They were given the options Definitely!, Nope or I didn't manage to learn the skills, but I'm sure they would've been useful. A hundred percent of participants selected Definitely! which indicates the online course successfully met its outcomes.

During the open-ended questions most of the participants felt positive about their experience and felt that it met their expectations with one participant saying: "Gagne's theory of learning instruction is one of the things I am going to use, I can already see how it is going to help my students to deal with difficult concepts in microbiology. This theory not only allows you to unpack the concepts, but it gives me the opportunity to determine if students are learning. Thinking about threshold concepts in my field I can already see how I am going to unpack them to my students, I am excited, I can't wait to do this!" another said: "When I heard "101" I thought it would be a lot more basic and perhaps a waste of time. I was pleasantly surprised!'.

\section{Conclusion}

This paper aimed to show that an online short course can help lecturers at a university of technology gain the skills necessary to operate the LMS in a way that allows students to reach the intended outcomes. The results showed that participants of the online short course had a positive experience and felt that they were better able to communicate, navigate and build content on the LMS. Of the 63 graduates of 5 iterations of the online short course, 33 completed the feedback survey. These results showed that $84 \%$ felt that they can 
communicate via announcements, $85 \%$ via email and $79 \%$ via discussion boards. When navigating the LMS, $82 \%$ were able to customize their personal settings after the course, $63 \%$ use the groups feature and $85 \%$ edit content using the formatting box. During the content building skills section, $94 \%$ of participants felt very positive toward adding files as well as images $(85 \%)$ and videos $(82 \%)$. Only $57 \%$ of participants felt that they have mastered the blogs tool which indicates an area for improvement.

This online short course assists the e-learning department in training lecturers in the use of the LMS and other educational technologies especially as it caters for different time schedules and geographical locations. There is also no limit on the number of participants so more lecturers can be trained at a time than the current method where the capacity of the training venue determines the number of participants. The course also provides participants with a social network which is important as we are social creatures (Camarero Cano, 2015).

A follow up to this course that delves deeper into the more intermediate functions of the LMS can help further train lecturers to level up their blended and online learning skills especially the online assessment skills.

\section{References}

Beatty, B., \& Ulasewicz, C. (2006). Faculty perspectives on moving from blackboard to the moodle learning management system. TechTrends, 50(4), 36-45.

Bolarinwa, O. A. (2015). Principles and methods of validity and reliability testing of questionnaires used in social and health science researches. Nigerian Postgraduate Medical Journal, 22(4), 195-201.

Camarero Cano, L. (2015). Techno-social communities. Evolution of analog communication towards interaction analog-digital. Mediterranean Journal of Communication, 6(1), 187-195.

De Vos, A., Strydom, H., Fouche, C. B., \& Delport, C. S. (2011). Research at grass roots: For social sciences and human service professions. Pretoria: Van Schaik Publishers.

Foster, C. W. (2016). The lived persistent meaning of abrupt, permanent separation from a black, female nanny during early childhood: A phenomenological psychological study. Saybrook University.

Lee, W. W., \& Owens, D. L. (2000). Multimedia-based instructional design. San Francisco : Jossey-Bass/Pfeiffer.

McGill, T. J., \& Klobas, J. E. (2009). A task-technology fit view of learning management system impact. Computers \& Education, 52(2), 496-508.

Osuna-Acedo, S., \& Gil-Quintana, J. (2017). The European project ECO. Breaking barriers in the access to knowledge. Education XXI, 20(2), 189-213.

Pullman, E., \& Graham, C. R. (2018). Comparing K-12 online and blended teaching competencies: a literature review. Distance Education, 39(3), 411-432.

Stevens, K. B. (2013). Contributing factors to a successful online course development process. The Journal of Continuing Higher Education, 61(1), 2-11. 